\title{
A Two-Stage Adaptive Algorithm in the Frequency Domain for a Multichannel Feedforward Active Noise Control System
}

\author{
$\operatorname{Min} \mathbf{Z h u}{ }^{\dagger}$ \\ Computer Center, East China Normal University, Zhongshan North Rd., Shanghai, 200062, P.R. China \\ System Science and Technology, Akita Prefectural University, Yurihonjo, 015-0055, Japan \\ Huigang Wang \\ College of Marine, Northwestern Polytechnical University, Xi'an, Shaanxi, 710072, P.R. China \\ System Science and Technology, Akita Prefectural University, Yurihonjo, 015-0055, Japan
}

Guoyue Chen

System Science and Technology, Akita Prefectural University, Yurihonjo, 015-0055, Japan

\author{
Kenji Muto \\ College of Engineering, Shibaura Institute of Technology, Tokyo, 135-8548, Japan
}

(Received 8 June 2012; revised February and April of this year; accepted 25 April 2013)

The reference paths between original sources and reference sensors in multichannel feedforward active noise control (ANC) systems are often ignored by most ANC algorithms. Therefore, a two-stage adaptive algorithm in the frequency domain is proposed to deal with several of the more complicated cases, specifically addressing instances when the reference sensors must be placed far away from the noise sources. This two-stage system includes a blind pre-processing system cascaded with a traditional MFxLMS system; both systems operate in the frequency domain. The new algorithm can eliminate the effect of reference paths by performing blind pre-processing independently. The MFxLMS algorithm in the following stage can achieve faster convergence than the traditional algorithm without blind pre-processing. The computational complexity of the proposed algorithm is analysed and a numerical simulation using impulse responses measured in a real reverberant room is performed to verify the convergence performance of the proposed algorithm.

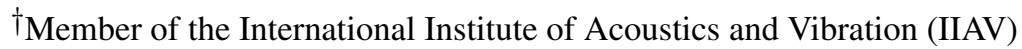

\section{INTRODUCTION}

Multichannel feedforward active noise control (ANC) systems have many potential applications in aircrafts, engines, and mechanics. Many successful algorithms, such as the multichannel filtered-x LMS (MFxLMS) algorithm, have been developed; these algorithms are summarized in the work of Elliot, ${ }^{1}$ and Haykin. ${ }^{2}$ The convergence performance of the MFxLMS algorithm, however, is limited by the properties of the reference signals and the structure of the secondary paths, phenomena that have been analysed in past work. ${ }^{3-7}$ Most multichannel ANC algorithms operate in the time domain; however, their computational complexity appears to increase with the length of the controller. A fast and efficient MFxLMS algorithm that operates in the frequency domain is also desirable and some block filter-x LMS algorithms for single channel or multichannel systems have been proposed to improve the computational efficiency of ANC systems with fast Fourier transform (FFT). ${ }^{14-16}$

Most existing algorithms are focused on the online or offline modelling and estimation of secondary paths; however, reference paths between the original sources and the reference sensors are often ignored because the reference sensors are assumed to be located close to the original noise sources. In many practical applications, the reference sensors cannot be placed close to the original noise sources because of physical environmental limitations. Therefore, because of the lack of reference paths, the data received by the reference sensors has a high correlation each other. A simple example can be used to explain the effect of the reference paths. If all of the original sources are uncorrelated noise, the data received by the reference sensors will be the combination of the original noise sources via their respective reference paths. Thus, the correlation properties of the reference signals are completely determined by the reference paths. A detailed theoretical analysis of the effect of reference paths and secondary paths on the performance of the MFxLMS algorithm in the frequency domain was presented in our previous paper. ${ }^{9}$

Elliott ${ }^{8}$ proposed an optimal controller in the frequency domain to improve the MFxLMS convergence by including prior information about the reference signals-namely, the cross 